\title{
The genus Cyphostemma (Planch.) Alston (Vitaceae) in Angola
}

\author{
Filipe de Sousa ${ }^{1}$, Estrela Figueiredo ${ }^{2}$ and Gideon F. Smith ${ }^{3}$ \\ ${ }^{1}$ Department of Plant and Environmental Sciences, University of Gothenburg, Box 461, SE-40530 Göteborg, \\ Sweden (email: filipedeportugal@gmail.com) (corresponding author). \\ 2 Department of Botany, P.O.Box 77000, Nelson Mandela Metropolitan University, Port Elizabeth, 6031 \\ South Africa / Centre for Functional Ecology, Departamento de Ciências da Vida, Universidade de Coimbra, \\ 3001-455 Coimbra, Portugal (email: estrelafigueiredo@ hotmail.com). \\ ${ }^{3}$ Office of the Chief Director: Biosystematics Research \& Biodiversity Collections, South African National \\ Biodiversity Institute, Private Bag X101, Pretoria, 0001 South Africa / H.G.W.J. Schweickerdt Herbarium, \\ Department of Plant Science, University of Pretoria, Pretoria, 0002 South Africa and Centre for Functional \\ Ecology, Departamento de Ciências da Vida, Universidade de Coimbra, 3001-455 Coimbra, Portugal \\ (email: g.smith@sanbi.org.za).
}

Summary: An overview of the 22 taxa recorded in the genus Cyphostemma (Planch.) Alston (Vitaceae) in Angola is presented. An identification key to all taxa recorded is provided, together with a referenced list of taxa with synonymy, geographical distribution range, endemic status and the citation of type specimens that originated from the country. Distribution maps are also presented. A list of herbarium collections consulted is given in an appendix.

Zusammenfassung: Es wird eine Übersicht über die 22 aus Angola bekannten Taxa der Gattung Cyphostemma (Planch.) Alston (Vitaceae) gegeben. Der Beitrag besteht aus einem Schlüssel zu allen Taxa, sowie einer referenzierten Taxonliste mit Synonymen, geographischer Verbreitung (inkl. Verbreitungskarten), Angaben zum Endemiestatus und Zitaten der Typusbelege, soweit sie aus dem Land stammen. Ein Anhang listet die untersuchten Herbarbelege auf.

\section{Introduction}

The family Vitaceae, which includes Vitis vinifera L., the source of table and wine grapes, consists of just over 700 species and 13 genera, making this a rather small family on global standards (Mabberley, 2008). Most species are woody vines with branched tendrils, but large, fat-stemmed species are also included in some genera. Flowers are rather small and insignificant, while the leaves are generally simple and deeply invaginated, or compound, and known for their striking autumn colours. The fruit of some representatives of the Vitaceae can be poisonous. The Vitaceae in An- gola were treated for Conspectus florae angolensis (Exell \& Mendonça, 1954) and more recently enumerated for Plants of Angola by Retief (2008). These treatments recognised five Vitaceae genera occurring naturally in the country [Ampelocissus Planch., Cayratia Juss., Cissus L., Cyphostemma (Planch.) Alston and Rhoicissus Planch.], while Vitis vinifera L. was recorded as having escaped from cultivation. The genus Cyphostemma (Planch.) Alston, previously included in Cissus L. as sect. Cyphostemma Planch. (Planchon, 1887), was elevated to the rank of genus by Alston (1931), a classification adopted by Descoings (1960). Descoings (1960) made several combinations in Cyphostemma that were invalid because the basionym was not clearly cited (ICBN Art. 33.2). Later, he published again the combinations (Descoings, 1967) in order to validate them, but meanwhile Wild \& Drummond (1966) had already unintentionally validated some of the names, therefore some names in Descoings' second set of combinations are also not accepted (being superfluous names). Cyphostemma comprises c. 150 species (Mabberley, 2008) and is widely distributed throughout tropical Africa. In Angola it is represented by 22 species, of which nine are endemic. Representatives of Cyphostemma differ from species included in the related genus Cissus, through having constricted flower buds and a nectary disk of four glands, which are free from each other (Descoings, 1960). The majority of species are erect, prostrate or climbing perennial herbs or shrubs, with or without tendrils (Wild \& Drummond, 1966). Several species of southern African Cyphostemma are thick-stemmed succulents or 
have succulent leaves, giving them horticultural interest among collectors (Smith et al., 1997; Figueiredo \& Smith, 2009).

\section{Material and methods}

The collections of Cyphostemma from Angola kept at the Herbaria of the Centro de Botannica (LISC) and University of Lisbon (LISU) in Lisbon, Portugal, and the National Herbarium of South Africa (PRE), in Pretoria, were examined. The Herbarium of the University of Coimbra (COI), Coimbra, Portugal, was contacted for permission to examine its specimens, but according to its curator it does not hold any collections of Cyphostemma from Angola. However, many specimens from COI are cited in the literature and types of Angolan Cyphostemma names from that herbarium are accessible in JSTOR (http://plants.jstor.org). These and other types were therefore examined on-line in JSTOR. The type material of names established in Cyphostemma for species that occur in Angola is indicated in the text, and for those specimens the herbarium barcode is given to facilitate searches using the JSTOR facility. Distribution maps were made using ArcGIS 9.3 from ESRI. Specimens cited in the literature that we did not examine were included in the maps using different symbols (open symbols). The full list of specimens used to prepare these maps is presented in Appendix 1.

\section{Results}

Twenty-two taxa of Cyphostemma are currently known to occur in Angola, following the recent recording of C. wittei (Staner) Wild \& R.B.Drumm. and C. congestum (Baker) Desc. ex Wild \& R.B.Drumm. as part of the flora of the country (Sousa et al., 2011). Two further possible records remain to be investigated when more material is available.

\section{List of taxa}

$\mathrm{H}=$ Habit; $\mathrm{D}=$ Distribution by province (provinces: BO-Bengo, BE-Benguela, BI-Bié, CA-Cabinda, CC-Cuando-Cubango, CN-Cuanza Norte, CS-Cuanza Sul, CU-Cunene, HAHuambo, HI-Huíla, LA-Luanda, LN-Lunda Norte, LS-Lunda Sul, MA-Malange, MO-Moxico, NA-Namibe, UI-Uíge, ZA-Zaire).

1. Cyphostemma adenocaule (Steud. ex A.Rich.) Desc. ex Wild \& R.B.Drumm., Fl. Zamb. 2: 473 (1966) subsp. adenocaule. Retief in Figueiredo \& Smith, Plants of
Angola: 169 (2008). Type from Ethiopia.

Cissus adenocaulis Steud. ex A.Rich., Tent. Fl. Abyss. 1: 111 (1847). Exell \& Mendonça, C.F.A. 2(1): 66 (1954).

Cyphostemma adenocaule (Steud. ex A.Rich.) Desc., Notul. Syst. (Paris) 16: 120 (1960), comb. inval.

$\mathrm{H}$ : Climbing herb with tuberous rootstock. D: BE, BO, CA, CN, CS, HI, LA, MA, NA, ZA. (Figure 1.)

2. Cyphostemma adenopodum (Sprague) Desc., Nat. Monspel., Sér. Bot. 18: 218 (1967). Retief in Figueiredo \& Smith, Plants of Angola: 169 (2008). Type from Uganda.

Cissus adenopoda Sprague, Bull. Misc. Inf. Kew 1906: 247 (1906). Exell \& Mendonça, C.F.A. 2(1): 62 (1954).

Cyphostemma adenopodum (Sprague) Desc., Notul. Syst. (Paris) 16: 120 (1960), comb. inval.

$\mathrm{H}$ : Climbing herb to several metres long from a tuberous rootstock. D: CA. (Figure 1.)

3. Cyphostemma chloroleucum (Welw. ex Baker) Desc. ex Wild \& R.B.Drumm., Fl. Zamb. 2,2: 466 (1966). Retief in Figueiredo \& Smith, Plants of Angola: 169 (2008). Figueiredo et al., Bothalia 39(2): 201 (2009). Type: Angola, entre Lago Ivantala e Quilengues, Welwitsch 1453 (BMBM000838246, LISU-LISU208728 \& LISU208730 \& LISU208729).

Vitis chloroleuca Welw. ex Baker in Oliver, Fl. Trop. Afr. 1: 406 (1868).

Cissus chloroleuca (Welw. ex Baker) Planch. in A. \& C. De Candolle, Mon. Phan. 5(2): 592 (1887); Exell \& Mendonça, C.F.A. 2(1): 60 (1954).

Cyphostemma chloroleucum (Welw. ex Baker) Desc., Notul. Syst. (Paris) 16: 120 (1960), comb. inval.

Vitis andongensis Welw. ex Baker in Oliver, Fl. Trop. Afr. 1: 406 (1868).

Cyphostemma andongensis (Welw. ex Baker) Desc., Nat. Monspel., Sér. Bot. 18: 218 (1967). Type: Angola, Pungo Andongo, Pedra de Cambondo, Welwitsch 1463 (B†?, BMBM000838248 \& BM000838247, COI-COI00005280, K, LISU-LISU208726 \& LISU208727).

Cissus hypargyrea Gilg in Warburg, KuneneSambesi-Expedition: 293 (1903).

Cyphostemma hypargyreum (Gilg) Desc., Nat. Monspel., Sér. Bot. 18: 222 (1967). 


\section{Key to the species of Cyphostemma recorded for Angola}

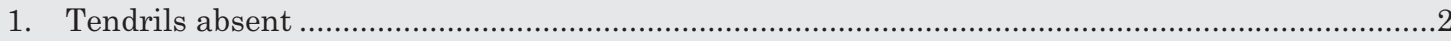

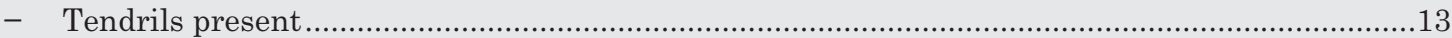

2. Plants with perennial, often massively thickened, succulent stem and papery bark...................

- Plants without a thickened, succulent stem and papery bark ................................................5

3. Stem squat, sub-spherical; leaflets sub-orbicular.................................................................. uter

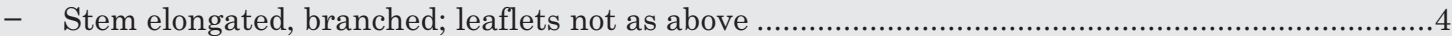

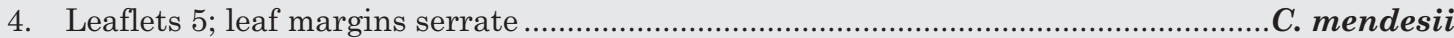

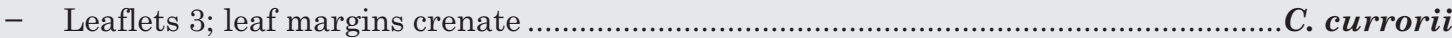

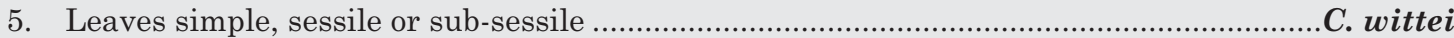

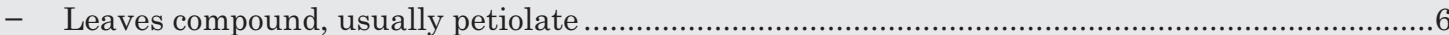

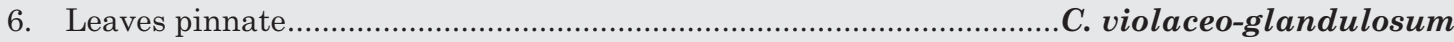

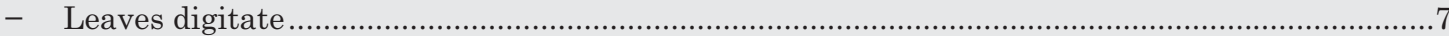

7. Leaflets oblong-lanceolate to linear, glabrous, glabrescent or pubescent .................................

- Leaflets cordate-ovate, obovate-elliptical to oblanceolate, never glabrous...................................11

8. Stipules large (up to $22 \times 16 \mathrm{~mm}$ ), cordate, amplexicaul .............................. grandistipulatum

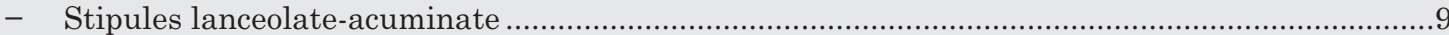

9. Stems and leaves glabrous, stipules usually 2 or more $\mathrm{cm}$ long; inflorescence $10-20 \mathrm{~cm}$ wide ...... C. junceum subsp. jatrophoides

- Stems and leaves not glabrous, stipules up to $1.5 \mathrm{~cm}$ long; inflorescence up to $6 \mathrm{~cm}$ wide...........10

10. Distal leaves sessile; leaflets serrate-crenate, usually with glandular hairs on the nerves abaxially; inflorescence smaller than subtending leaf.

C. stenolobum

- Distal leaves petiolate; leaflets entire to serrate-dentate, sub-glabrous; inflorescence up to $5 \mathrm{~cm}$ wide

C. fugosioides

11. Plant densely pilose-glandulose from the base

C. pruriens

- Plant pubescent but never densely pilose-glandulose

12. Leaves densely ferruginous-tomentose below; inflorescence branches glandular

C. chloroleucum

- Leaves never densely ferruginous-tomentose below; inflorescence branches quite eglandular.

13. Leaves pedately arranged

C. luteum

- Leaves digitately arranged.

C. adenocaule

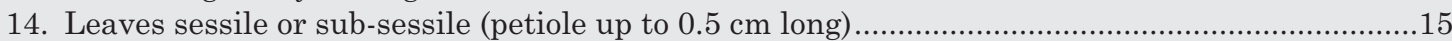

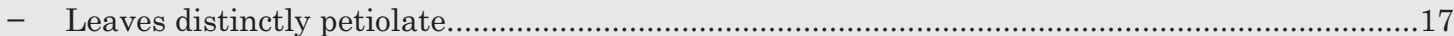

15. Inflorescence branches, calyx and corolla glabrous or puberulous; leaflets ovate-elliptic, up to 5.5 $\times 2 \mathrm{~cm}$

C. subciliatum

- Inflorescence branches, calyx and corolla densely white pubescent; leaflets elliptic-lanceolate, up to $14 \times 5 \mathrm{~cm}$

16. Leaflets glabrous, up to $10 \times 3 \mathrm{~cm}$, undulate to slightly serrate

- Leaflets puberulous to pubescent, up to $14 \times 5 \mathrm{~cm}$, distinctly dentate to serrate ....C. congestum

17. Stem densely covered with glandular hairs to $5 \mathrm{~mm}$ long....

C. pendulum

- Stem not as above 18

18. Leaflets broadly elliptic to sub-orbicular, apex rounded

C. ruacanense

- Leaflets ovate, elliptic or lanceolate, apex acute to acuminate

19. Leaflets 3

- Leaflets 5

C. adenopodum

20. Inflorescence branches eglandular, densely white-pubescent

C. johannis

- Inflorescence branches glandular, densely to sparsely pubescent . .21

21. Flower buds glandular; petioles to $15 \mathrm{~cm}$ long; leaves pubescent on nerves below; stipules quite large, suborbicular-acuminate, up to $25 \times 20 \mathrm{~mm}$.

C. stipulaceum

- Flower buds eglandular; petioles to $5 \mathrm{~cm}$ long; leaves densely pubescent to tomentose below; stipules inconspicuous

C. crassiusculum 
Type:Angola, Kubango, Kabindere, $1150 \mathrm{~m}$, 1899/11/2, Baum 361 (B† holo-; BM, COICOI00005281, G-G00023853, K, WW19010006645 iso-).

Cissus decurrens Gilg \& M.Brandt, Bot. Jahrb. Syst. 46: 452, 504 (1911). Type: Angola, Chibemba (Gambos), Newton 230 (B†? holo-).

H: Herb up to $60 \mathrm{~cm}$ high. D: BE, CN, CS, CU, HI, MA. (Figure 2.)

4. Cyphostemma congestum (Baker) Desc. ex Wild \& R.B.Drumm., Fl. Zamb. 2(2): 473 (1966). Sousa et al., Bothalia 41(2): 260 (2011). Type from Nyasaland.

Vitis congesta Baker in Oliver, Fl. Trop. Afr. 1: 412 (1868).

Cyphostemma congestum (Baker) Desc., Notul. Syst. (Paris) 16: 120 (1960), comb. inval.

$\mathrm{H}$ : Climbing herb with tuberous root. D: CU. (Figure 2.)

5. Cyphostemma crassiusculum (Baker) Desc., Nat. Monspel., Sér. Bot. 18: 220 (1967). Retief in Figueiredo \& Smith, Plants of Angola: 169 (2008). Type: Angola, Loanda, entre Penedo e Conceição, Welwitsch 1495 (BMBM000838266, COI-COI00005288, K, LISULISU208737 \& LISU208738).
Vitis crassiuscula Baker in Oliver, Fl. Trop. Afr. 1: 406 (1868).

Cissus crassiuscula (Baker) Planch., Monogr. Phan. 5: 608 (1887). Exell \& Mendonça, C.F.A. 2(1): 68 (1954).

Cyphostemma crassiusculum (Baker) Desc., Notul. Syst. (Paris) 16: 120 (1960), comb. inval.

Cissus loandensis Gilg \& M.Brandt, Bot. Jahrb. Syst. 46: 537 (1912).

Cyphostemma loandense (Gilg \& M.Brandt) Desc., Nat. Monspel., Sér. Bot. 18: 224 (1967). Type: Angola, Luanda, Gossweiler 294 (B†? lectodesignated by Exell \& Mendonça (1954: 68).

$\mathrm{H}$ : Climber. D: BE, BO, CN, LA, MA, NA. Endemic. (Figure 3.)

6. Cyphostemma currorii (Hook.f.) Desc., Nat. Monspel., Sér. Bot. 18: 220 (1967). Retief in Figueiredo \& Smith, Plants of Angola: 169 (2008). Type: Moçâmedes, Baía dos Elefantes, Curror s.n. (Bł? iso-, K holo-).

Cissus currorii Hook.f., Niger Fl.: 265 (1849). Exell \& Mendonça, C.F.A. 2(1): 70 (1954).

Vitis currorii (Hook.f.) Baker in Oliver, Fl. Trop. Afr. 1: 401 (1868).

Cyphostemma currorii (Hook.f.) Desc., Notul. Syst. (Paris) 16: 121 (1960), comb. inval.

Cissus macropus Welw., J. Linn. Soc., Bot. 8 : 77 (1864).

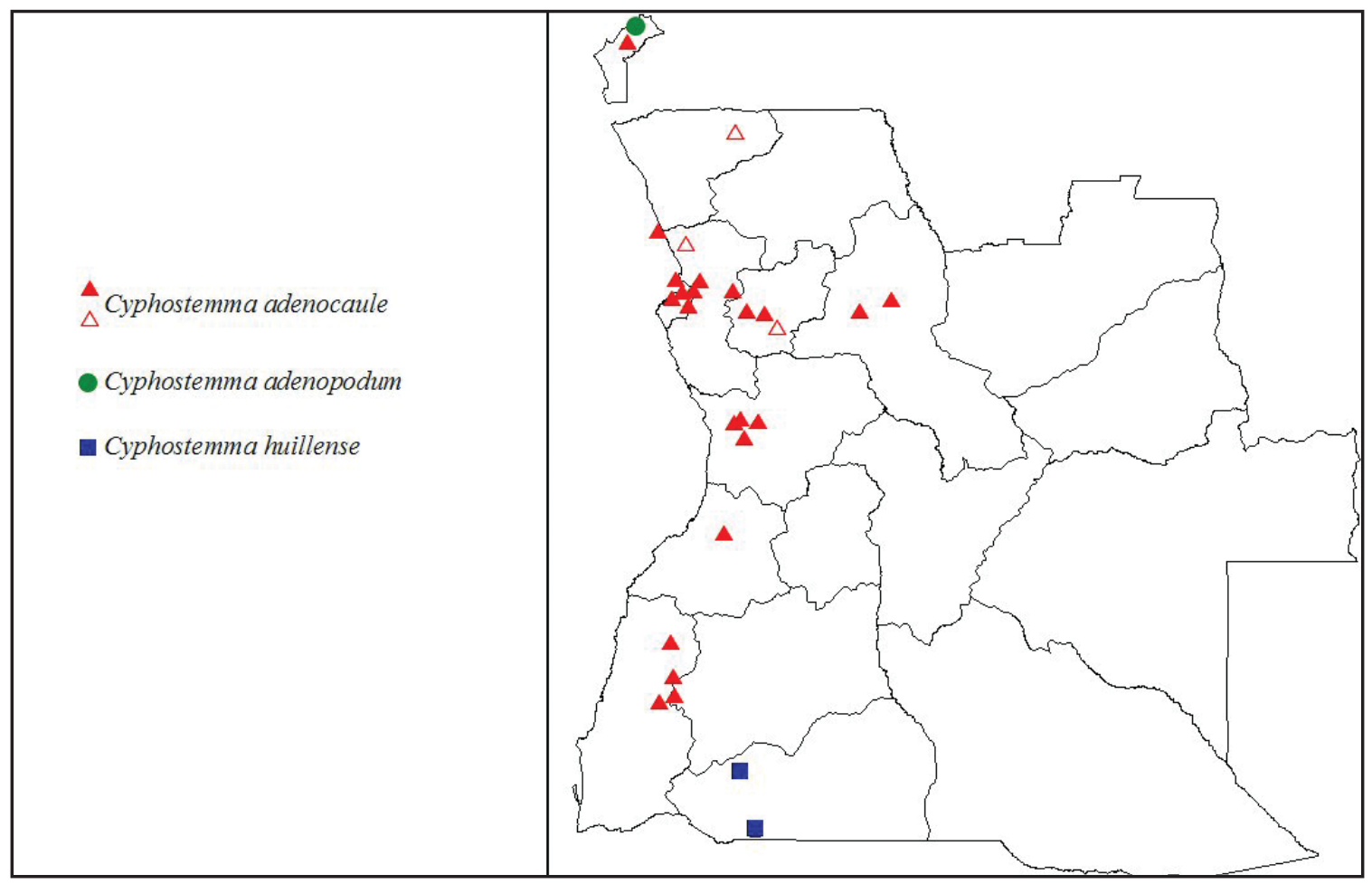

Figure 1. Geographical distribution map of C. adenocaule (Steud. ex A.Rich.) Desc. ex Wild \& R.B.Drumm., C. adenopodum (Sprague) Desc. and C. huillense (Exell \& Mendonça) Desc. in Angola. 

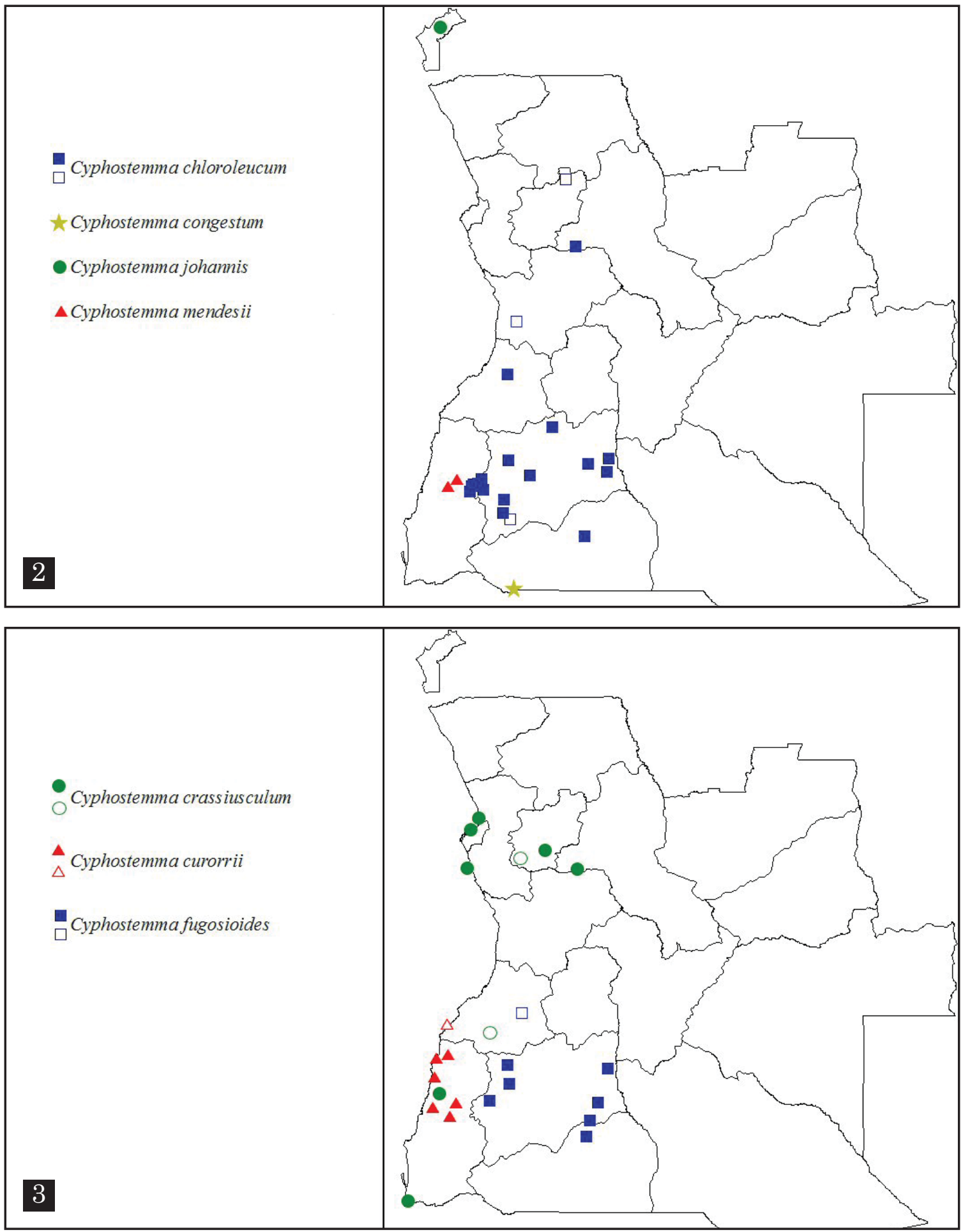

Figure 2. Geographical distribution map of C. chloroleucum (Welw. ex Baker) Desc. ex Wild \& R.B.Drumm., C. congestum (Baker) Desc. ex Wild \& R.B.Drumm., C. johannis (Exell \& Mendonça) Desc. and C. mendesii F.Sousa in Angola. Figure 3. Geographical distribution map of C. crassiusculum (Baker) Desc., C. currorii (Hook.f.) Desc. and C. fugosioides (Gilg) Desc. ex Wild \& R.B.Drumm. in Angola. 
Vitis macropus (Welw.) Baker in Oliver, Fl. Trop. Afr. 1: 405 (1868).

Cyphostemma macropus (Welw.) Desc., Notul. Syst. (Paris) 16: 122 (1960), comb. inval. Cyphostemma macropus (Welw.) Desc., Nat. Monspel., Sér. Bot. 18: 224 (1967). Type: Angola, Moçâmedes, Welwitsch 1446 (BM, LISULISU208739 \& LISU208740 \& LISU208741).

$\mathrm{H}$ : Pachycaul trees to $6 \mathrm{~m}$ tall, along desert coastline. D: BE, NA. (Figures 3-6.)

7. Cyphostemma fugosioides (Gilg) Desc. ex Wild \& R.B.Drumm., Fl. Zamb. 2(2): 469 (1966). Retief in Figueiredo \& Smith, Plants of Angola: 169 (2008). Figueiredo et al., Bothalia 39(2): 201 (2009). Type: Angola, Chitanda, 1150 m, 1899/9/27, Baum 174 (B† holo-; BM, COI-COI00005289, G-G00023854, K, MM0109127, W-W19010009447 iso-).

Cissus fugosioides Gilg in Warburg, KuneneSambesi-Expedition: 294 (1903); Exell \& Mendonça, C.F.A. 2(1): 57 (1954).

Cyphostemma fugosioides (Gilg) Desc., Notul. Syst. (Paris) 16: 121 (1960), comb. inval.

$\mathrm{H}$ : Herb up to $50 \mathrm{~cm}$ tall, with a tuberous rootstock. D: BE, CU, HI. (Figure 3.)

8. Cyphostemma grandistipulatum (Gilg \& M.Brandt) Desc., Nat. Monspel., Sér. Bot. 18: 222 (1967). Retief in Figueiredo \& Smith, Plants of Angola: 169 (2008). Type: Angola, Huíla, Antunes 110 (B†? lecto- designated by Exell \& Mendonça (1954: 56)).

Cissus grandistipulata Gilg \& M.Brandt, Bot. Jahrb. Syst. 46: 491 (1912). Exell \& Mendonça, C.F.A. 2(1): 56 (1954).

Cyphostemma grandistipulatum (Gilg \& M.Brandt) Desc., Notul. Syst. (Paris) 16: 121 (1960), comb. inval.

H: Succulent? herb up to $90 \mathrm{~cm}$ high. D: HI. (Figure 7.)

9. Cyphostemma huillense (Exell \& Mendonça) Desc., Nat. Monspel., Sér. Bot. 18: 222 (1967). Retief in Figueiredo \& Smith, Plants of Angola: 169 (2008). Type: Angola, Huíla, Morros de Cualeque [Calueque], 1000-1100 m, 1937, Exell \& Mendonça 2708 (BM holo-; COICOI00005292 \& COI00005291, LISCLISC001323, iso-).

Cissus huillensis Exell \& Mendonça, Bol. Soc. Brot. 26: 229 (1952). Exell \& Mendonça, C.F.A. 2(1): 62 (1954).

H: Climber. D: CU. Endemic. (Figure 1.)

10. Cyphostemma johannis (Exell \& Mendonça) Desc., Nat. Monspel., Sér. Bot. 18: 222 (1967).
Retief in Figueiredo \& Smith, Plants of Angola: 169 (2008). Type: Angola, Cabinda, Maiombe, Buco Zau, 1916/10, Gossweiler 7238 (BM-BM000838236 p.p. holo-; LISCLISC001325 \& LISC001324, LISU p.p. iso-).

Cissus johannis Exell \& Mendonça, Bol. Soc. Brot. 26: 231 (1952). Exell \& Mendonça, C.F.A. 2(1): 64 (1954).

Cyphostemma johannis (Exell \& Mendonça) Desc., Notul. Syst. (Paris) 16: 122 (1960), comb.inval.

H: Climber. D: CA. Endemic. (Figure 2.)

11. Cyphostemma junceum (Webb) Desc. ex Wild \& R.B. Drumm., Fl. Zamb. 2(2): 465 (1966) subsp. jatrophoides (Welw. ex Baker) Verdc., Fl. Trop. E. Africa Vitac.: 62 (1993). Retief in Figueiredo \& Smith, Plants of Angola: 169 (2008). Type: Angola, Ambaca, entre os Rios Lutete e Zamba, Welwitsch 1474 (B十?, BM-BM000838275, COI-COI00005293, LISULISU208718 \& LISU208719 \& LISU208720 \& LISU208721).

Vitis jatrophoides Welw. ex Baker in Oliver, Fl. Trop. Afr. 1: 400 (1868) pro parte excl. spec Barter.

Cissus jatrophoides (Welw. ex Baker) Planch. in A. \& C. De Candolle, Mon. Phan. 5(2): 579 (1887); Exell \& Mendonça, C.F.A. 2(1): 57 (1954).

Cyphostemma jatrophoides (Welw. ex Baker) Desc., Notul. Syst. (Paris) 16: 122 (1960), comb. inval.

Cyphostemma jatrophoides (Welw. ex Baker) Desc., Nat. Monspel., Sér. Bot. 18: 226 (1967).

Cissus chlorantha Gilg in Warburg, Kunene-Sambesi-Expedition: 295 (1903). Type: Angola, Kubango, Chirumbu, 1200 m, 1899/10/14, Baum 278 (B† holo-; GG00023856, W-W19010009245 iso-).

H: Herb up to $1.3 \mathrm{~m}$ high, with a tuberous rootstock. D: BE, BI, CN, CU, HA, HI, LS. (Figure 8.)

Note: Welwitsch $1474(\mathrm{~K})$ is Cissus petiolata Hook.f.

12. Cyphostemma luteum (Exell \& Mendonça) Desc., Nat. Monspel., Sér. Bot. 18: 224 (1967). Retief in Figueiredo \& Smith, Plants of Angola: 169 (2008). Type: Angola, Bié, Rio Cubango, Vila da Ponte, Gossweiler 2355 (BMBM000838219 holo-, LISC-LISC001326 iso-).

Cissus lutea Exell \& Mendonça, Bol. Soc. Brot. 26: 228 (1952). Exell \& Mendonça, C.F.A. 2(1): 58 (1954).

Cyphostemma luteum (Exell \& Mendonça) Desc., Notul. Syst. (Paris) 16: 122 (1960), comb. inval.

$\mathrm{H}$ : Herb with multi-headed rootstock. D: HI. Endemic. (Figure 9.) 

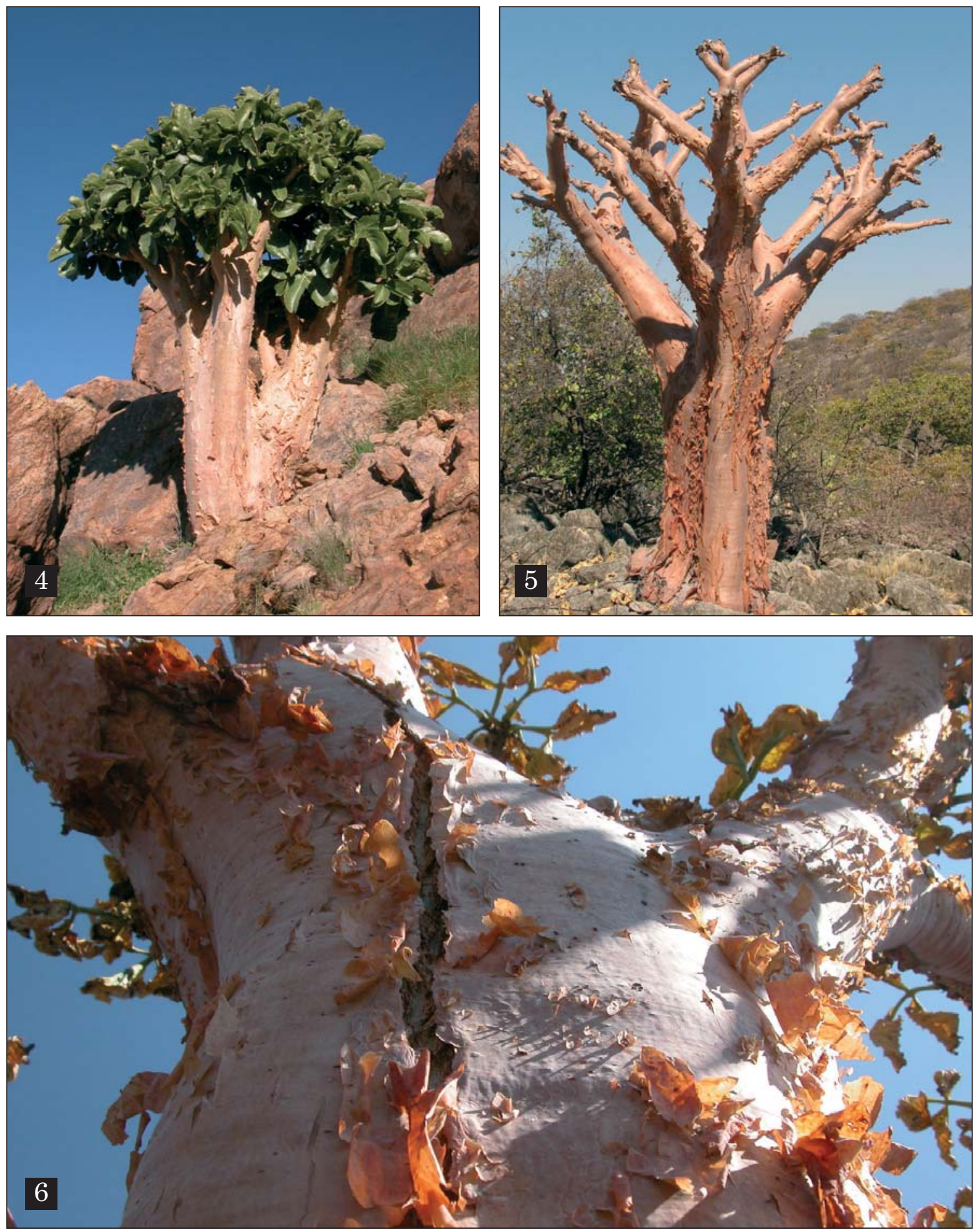

Figure 4. C. currorii in leaf. Photo taken in Namibia on the Groot Spitzkoppe on 4 May 2005 by Tim Harvey. Figure 5. C. currorii in the dry season. Photo taken in Namibia at Baobab Bend, approximately $40 \mathrm{~km}$ south of Opuwo on the C43, on 24 July 2007 by Tim Harvey. Figure 6. Close-up of the bark of $C$. currorii. Photo taken in Namibia at Baobab Bend, approximately $40 \mathrm{~km}$ south of Opuwo on the C43, on 10 May 2005 by Tim Harvey. 

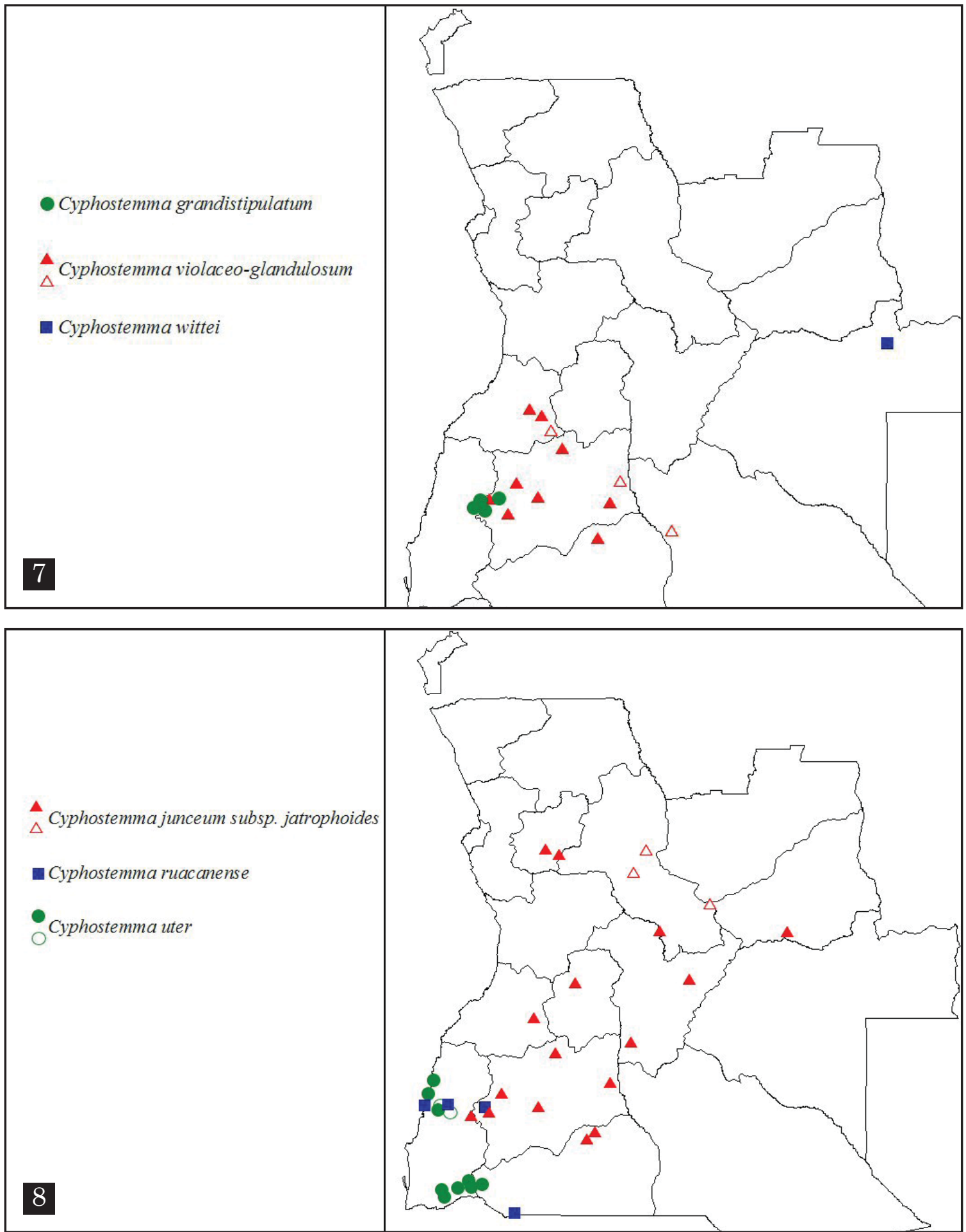

Figure 7. Geographical distribution map of C. grandistipulatum (Gilg \& M.Brandt) Desc., C. violaceoglandulosum (Gilg) Desc. and C. wittei (Staner) Wild \& R.B.Drumm. in Angola. Figure 8. Geographical distribution map of $C$. junceum (Webb) Desc. ex Wild \& R.B. Drumm. subsp. jatrophoides (Welw. ex Baker) Verdc., C. ruacanense (Exell \& Mendonça) Desc. and C. uter (Exell \& Mendonça) Desc. in Angola. 

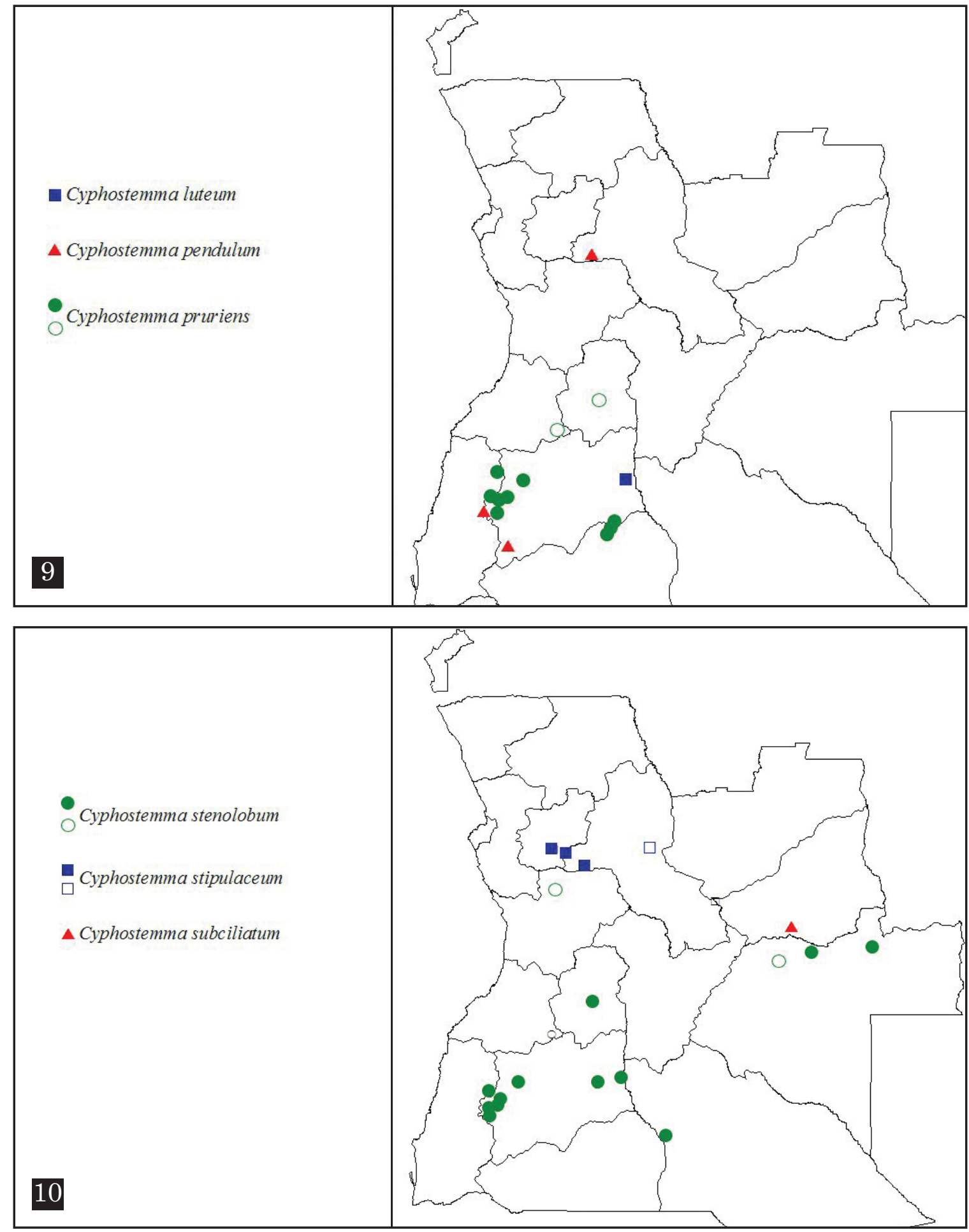

Figure 9. Geographical distribution map of C. luteum (Exell \& Mendonça) Desc., C. pendulum (Welw. ex Baker) Desc. and C. pruriens (Welw. ex Baker) Desc. in Angola. Figure 10. Geographical distribution map of C. stenolobum (Welw. ex Baker) Desc. ex Wild \& R.B.Drumm., C. stipulaceum (Baker) Desc. and C. subciliatum (Baker) Desc. ex Wild \& R.B.Drumm. in Angola. 
13. Cyphostemma mendesii F.Sousa, Phytotaxa 7: 36 (2010). Type: Angola, Moçâmedes, Dois Irmãos, ca. Pedra da Providência, 21 December 1955, Mendes 1145 (holo- \& iso- LISC).

H: Rhizomatous herb with succulent stems. D: NA. Endemic. (Figure 2.)

14. Cyphostemma pendulum (Welw. ex Baker) Desc., Nat. Monspel., Sér. Bot. 18: 226 (1967). Retief in Figueiredo \& Smith, Plants of Angola: 169 (2008). Type: Angola, Pungo Andongo, Presídio, Welwitsch 1472 (BMBM000838242, COI-COI00005296, K, LISULISU208731 \&, LISU208734 \& LISU208733 \& LISU208732).

Vitis pendula Welw. ex Baker in Oliver, Fl. Trop. Afr. 1: 407 (1868).

Cissus pendula (Welw. ex Baker) Planch. Monogr. Phan. 5: 594 (1887). Exell \& Mendonça, C.F.A. 2(1): 62 (1954).

Cyphostemma pendulum (Welw. ex Baker) Desc., Notul. Syst. (Paris) 16: 123 (1960), comb. inval.

$\mathrm{H}$ : Climbing herb to $90 \mathrm{~cm}$ long. D: HI, MA, NA. Endemic. (Figure 9.)

15. Cyphostemma pruriens (Welw. ex Baker) Desc., Nat. Monspel., Sér. Bot. 18: 226 (1967). Retief in Figueiredo \& Smith, Plants of Angola: 169 (2008). Type: Angola, Huíla, pr. Lopolo, Welwitsch 1454 (BM-BM000838250, LISU-LISU208717 \& LISU208716).

Vitis pruriens Welw. ex Baker in Oliver, Fl. Trop. Afr. 1: 408 (1868).

Cissus pruriens (Welw. ex Baker) Planch., Monogr. Phan. 5: 595 (1887). Exell \& Mendonça, C.F.A. 2(1): 57 (1954).

Cyphostemma pruriens (Welw. ex Baker) Desc., Notul. Syst. (Paris) 16: 123 (1960), comb. inval.

$\mathrm{H}$ : Erect or climbing herb up to $90 \mathrm{~cm}$ long. D: BE, CU, HA, HI, NA. Endemic. (Figure 9.)

16. Cyphostemma ruacanense (Exell \& Mendonça) Desc., Nat. Monspel., Sér. Bot. 18: 227 (1967). Retief in Figueiredo \& Smith, Plants of Angola: 169 (2008). Type: Angola, Huíla, Ruacaná, Rio Cunene, 1000 m, 9 June 1937, Exell \& Mendonça 2769 (BM-BM000838245 holo-; COI-COI00005297, LISC-LISC001329 \& LISC001328, iso-).

Cissus ruacanensis Exell \& Mendonça, Bol. Soc. Brot. 26: 229 (1952). Exell \& Mendonça, C.F.A. 2(1): 60 (1954).

Cyphostemma ruacanense (Exell \& Mendonça) Desc., Notul. Syst. (Paris) 16: 124 (1960), comb. inval.

H: Climber. D: CU, NA. (Figure 8.)

17. Cyphostemma stenolobum (Welw. ex Baker) Desc. ex Wild \& R.B.Drumm., Fl. Zamb. 2(2):
471 (1966). Retief in Figueiredo \& Smith, Plants of Angola: 169 (2008). Type: Angola, entre Lago Ivantala e Quilengues, Welwitsch 1449 (BM-BM000838265, COI-COI00005299, K, LISU-LISU208722 \& LISU208724 \& LISU208723 \& LISU208725).

Vitis stenoloba Welw. ex Baker in Oliver, Fl. Trop. Afr. 1: 408 (1868) pro parte excl. Welwitsch 1451.

Cissus stenoloba (Welw. ex Baker) Planch. in A. \& C. De Candolle, Mon. Phan. 5(2): 578 (1887); Exell \& Mendonça, C.F.A. 2(1): 58 (1954).

Cyphostemma stenolobum (Welw. ex Baker) Desc., Notul. Syst. (Paris) 16: 124 (1960), comb. inval.

$\mathrm{H}$ : Herb up to $1 \mathrm{~m}$ tall, from a narrowly elongated, tuberous rootstock. D: BE, CC, CS, HA, HI, MA, MO. (Figure 10.)

18. Cyphostemma stipulaceum (Baker) Desc., Nat. Monspel., Sér. Bot. 18: 228 (1967). Retief in Figueiredo \& Smith, Plants of Angola: 169 (2008). Type: Cuanza Norte, Cazengo, R. Luinha, Welwitsch 1490 (B†?, BMBM000838244, COI-COI00005300, LISULISU208735 \& LISU208736).

Vitis stipulacea Baker in Oliver, Fl. Trop. Afr. 1: 408 (1868).

Cissus stipulacea (Baker) Planch., Monogr. Phan. 5: 597 (1887). Exell \& Mendonça, C.F.A. 2(1): 64 (1954).

Cyphostemma stipulaceum (Baker) Desc., Notul. Syst. (Paris) 16: 125 (1960), comb. inval.

H: Climber. D: CN, MA. Endemic. (Figure 10.)

19. Cyphostemma subciliatum (Baker) Desc. ex Wild \& R.B.Drumm., Fl. Zamb. 2: 474, t. 97a (1966). Retief in Figueiredo \& Smith, Plants of Angola: 169 (2008). Type from Mozambique.

Vitis subciliata Baker in Oliver, Fl. Trop. Afr. 1: 409 (1868).

Cissus subciliata (Baker) Planch. in A. \& C. De Candolle, Monogr. Phan. 5(2): 594 (1887).

Cyphostemma subciliatum (Baker) Desc., Notul. Syst. (Paris) 16: 125 (1960), comb. inval.

Cissus marionae Exell \& Mendonça, Bol. Soc. Brot. ser. 2, 26: 230 (1952). Exell \& Mendonça, C.F.A. 2(1): 62 (1954).

Cyphostemma marionae (Exell \& Mendonça) Desc., Nat. Monspel., Sér. Bot. 18: 224 (1967). Type from Zambia.

$\mathrm{H}$ : Climbing herb with a tuberous, napiform root. D: LS. (Figure 10.)

20. Cyphostemma uter (Exell \& Mendonça) Desc., Nat. Monspel., Sér. Bot. 18: 229 (1967). Retief in Figueiredo \& Smith, Plants of Angola: 169 (2008). Type: Angola, Moçâmedes, 

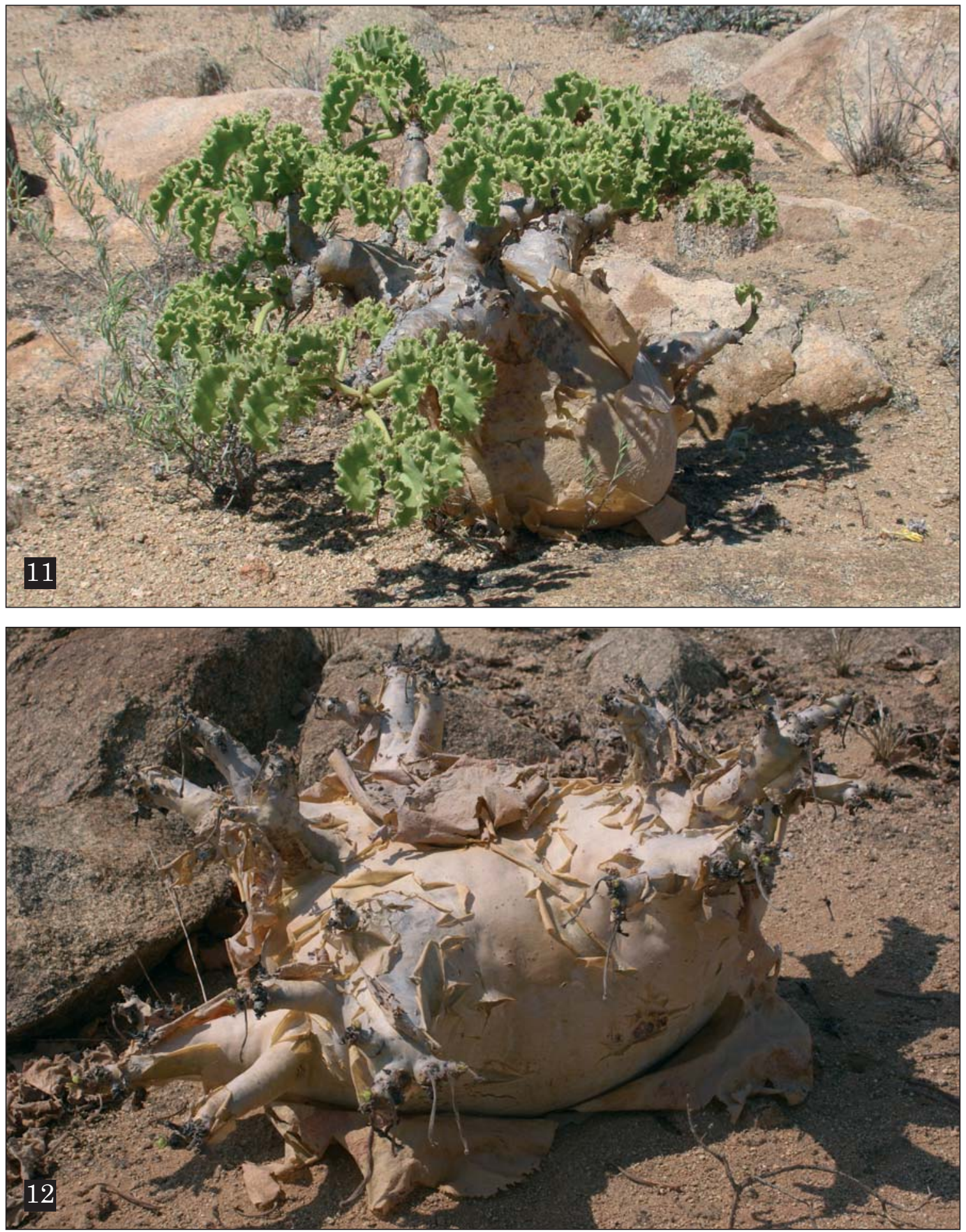

Figure 11. C. uter in leaf in the rainy season. Photo taken approximately $15 \mathrm{~km}$ east of the port city of Namibe in southern Angola on 14 January 2009 by Ian Oliver. Figure 12. C. uter in the dry season. Photo taken along the road from Leba Pass en route to Namibe in southern Angola on 6 August 2010 by Gideon F. Smith. 
Providência, 24 October 1937, Abreu 51 (BMBM000838241 iso-; COI-COI00005303 holo-).

Cissus uter Exell \& Mendonça, Bol. Soc. Brot., ser. 2, 26: 231-232 (1952). Exell \& Mendonça, C.F.A. 2(1): 70 (1954).

Cyphostemma uter (Exell \& Mendonça) Desc., Notul. Syst. (Paris) 16: 125 (1960), comb. inval.

$\mathrm{H}$ : Deciduous, caudiciform succulent to $1.5 \mathrm{~m}$ tall, in desert coastline. D: CU, NA. (Figures 8, 11-12.)

\section{Cyphostemma violaceo-glandulosum} (Gilg) Desc., Nat. Monspel., Sér. Bot. 18: 230 (1967). Figueiredo et al., Bothalia 39(2): 201 (2009). Retief in Figueiredo \& Smith, Plants of Angola: 169 (2008). Type: Angola, Kubango, Chirumbu, 1899/10/11, Baum 270 (B†, lectodesignated by Exell \& Mendonça (1954: 56); BM, COI-COI00005305, G-G00023926, K, MM0109115, S-S-G-1413 isolecto-).

Cissus violaceo-glandulosa Gilg in Warburg, Kunene-Sambesi-Expedition: 294 (1903). Exell \& Mendonça, C.F.A. 2(1): 56 (1954).

Cyphostemma violaceo-glandulosum (Gilg) Desc., Notul. Syst. (Paris) 16: 125 (1960), comb. inval.

$\mathrm{H}$ : Prostrate herb from a fleshy, woody, multiheaded rootstock. D: BE, CC, CU, HI. Endemic. (Figure 7.)

22. Cyphostemma wittei (Staner) Wild \& R.B.Drumm., Kirkia 2: 141 (1961). Sousa et al., Bothalia 41(2): 260 (2011). Type from Congo (Katanga).

Cissus wittei Staner, Contr. Fl. Katanga, Suppl. 4: 49 (1932).

$\mathrm{H}$ : Prostrate or scrambling herb from a thick fusiform woody tap-root. D: MO. (Figure 7.)

\section{Insufficiently known taxa}

Cyphostemma sp. aff. vogelii. Referred to as Cissus sp. aff. vogelii by Exell \& Mendonça (1954: 66).

Cyphostemma sp. aff. fugosioides. A climber up to $3.5 \mathrm{~m}$ high, with tendrils, 5 -foliolate leaves with narrow leaflets.

\section{Acknowledgements}

Mr Ian Oliver, Curator of the Karoo Desert National Botanical Garden of South Africa, and Dr Tim Harvey of California, USA, are thanked for the use of images of C. uter and C. currorii, respectively. We thank Michele Walters for producing the distribution maps. The curators of LISC, LISU and PRE are thanked for facilitating access to their collections. Dr Christien Bredenkamp of SANBI is thanked for constructive criticism on the paper.

\section{References}

Alston , A.H.G. (1931). Supplement. In Trimen, H., A Hand-book to the Flora of Ceylon, part 6. London, Dulau \& Co.

Descoings, B. (1960). Un genre méconnu de Vitacées: compréhension et distinction des genres "Cissus" L. et "Cyphostemma" (Planch.) Alston. Notul. Syst. (Paris) 16: 113-125.

Descoings, B. (1967). Note rectificative à propos de la nomenclature des Cyphostemma (Vitacées). Nat. Monspel. Sér. Bot. 18: 217-230.

Exell, A.W. \& MendonçA, F.A. (1954). Vitaceae. Conspectus florae angolensis 2: 35-71. Junta de Investigações do Ultramar, Lisboa.

Figueiredo, E. \& Smith, G.F. (2009). The succulent flora of Angola. Haseltonia 15: 69-78.

Figueiredo, E., Soares, M., Seibert, G., Smith, G.F. \& FADEN, R.B. (2009). The botany of the Cunene-Zambezi Expedition with notes on Hugo Baum (1867-1950). Bothalia 39: 185211.

MABBERLEY, D.J. (2008). Mabberley's Plant-book: a portable dictionary of plants, their classifications and uses. 3rd ed. Cambridge University Press, Cambridge.

Planchon, J.E. (1887). Cissus. In Candolle, A.L.P.P. DE \& Candolle, A.C.P. De, Monographiae Phanerogamarum Prodromi nunc Continuato 5(2): 472-472.

Retief, E. (2008). Vitaceae. In Figueiredo, E. \& SMith, G.F. Plants of Angola/Plantas de Angola. Strelitzia 22: 168-169. South African National Biodiversity Institute, Pretoria.

SMith, G.F., VAN JAARSveld, E.J., ARNOLD, T.H., Steffens, F.E., Dixon, R.D. \& Retief J.A. (1997). List of southern African succulent plants. Umdaus Press, Pretoria.

Sousa, F., Figueiredo, E. \& Smith, G.F. (2011). Vitaceae. A new and an overlooked record of Cyphostemma (Planch.) Alston in Angola. Bothalia 41(2): 160-161.

WiLD, H. \& DRUmmond, R.B. (1966). Vitaceae. In LAUnert, E. (ed.), Flora Zambesiaca, 2(2): 439-492. Flora Zambesiaca Managing Committee, London.

\section{Appendix 1. List of specimens used to pre-} pare the maps

C. adenocaule subsp. adenocaule: Albano Rocha 52 (LISC!); Albano Rocha 64 (LISC!); Anchieta 44 (B COI LISU!); Araújo 64 (LISC!); Barbosa \& Correia 9121 (LISC!, PRE!); Beatriz 38 (COI); Büttner 99 (B); Cameira 324 (LISC!); Exell \& Mendonça 3105 (BM COI LISC!); Exell \& Mendonça 3191 (BM COI); M.E.F.A. 726 (LISC!); Mendes 499 (LISC!); Mendes 933 (LISC!); Mendes 
1155 (LISC!); Mendes 1238 (LISC!); Monteiro s.n. (K); Monteiro, Santos \& Murta 122 (LISC!); Monteiro, Santos \& Murta 166 (LISC!); Pearson 2345 (B K); Pearson 2749 (K); Raimundo, Matos \& Figueira 209 (LISC!); Santos 1377 (LISC!, PRE!); Teixeira 3689 (LISC!); Teixeira et al. 10298 (LISC!); Teixeira et al. 11133 (LISC!); Teixeira et al. 11498 (LISC!); Welwitsch 1452 (BM LISU!); Welwitsch 1479 (BM LISU!); Welwitsch 1479b (BM LISU!); Welwitsch 1492 (BM LISU!); Welwitsch 1494 (BM LISU!); Welwitsch 1493 (BM COI K LISU!).

C. adenopodum: Gossweiler 7690 (COI K LISC! LISU!); Gossweiler 8208 (COI LISU!).

C. chloroleucum: Anchieta 67 (COI LISU!); Anchieta 86 (COI LISU!); Baum 361 (B† BM COI G K W); Borges 318 (LISC!); Borges 318a (PRE!); Gossweiler 2217 (BM COI LISC!); Gossweiler 3649 (BM COI LISC); Gossweiler 3650 (COI LISC!); Gossweiler 8768 (BM); Gossweiler 9247 (BM COI K); Henriques 792 (LISC! LISU!); Mendes 197 (LISC!); Mendes 766 (LISC!); Mendes 880 (LISC!); Mendes 980 (LISC!); Mendes 1129 (LISC!); Menezes 1277 (LISC!); Menezes 1373 (LISC!); Menezes 2408 (LISC!); Menezes 3299 (LISC!); Menezes 3531 (LISC!, PRE!); Newton 230 (B†?); Santos 53 (LISC!); Santos 79 (LISC!); Teixeira 1468 (LISC!); Teixeira 1822 (LISC!); Welwitsch 1453 (BM LISU!); Welwitsch 1463 (B†? BM COI K LISU!).

C. congestum: Kotze 57 (PRE!); Rycroft 2423 (PRE!).

C. crassiusculum: Carrisso \& Sousa 334 (BM COI LISC!); Gossweiler 294 (B†?); Gossweiler 294c (BM); Gossweiler 1510 (BM COI); Gossweiler 5172 (LISC! LISU!); Gossweiler 5278 (BM LISC! LISU!); Gossweiler 8276 (BM K P); Henriques 65 (LISC!); Humbert 16134 (P); Monteiro, Santos \& Murta 121 (LISC!, PRE!); Thompson 20 (PRE!); Welwitsch 1469 (BM LISU!); Welwitsch 1492b (B BM COI K LISU!); Welwitsch 1495 (BM COI K LISU!).

C. currorii: Curror s.n. (Bł? K); Mendes 138 (LISC!); Mendes 424 (LISC!); Mendes 1224 (LISC!); Mendes 1733 (LISC!); Mendes 8262? (LISC!); Mendonça 4530 (LISC!); Menezes 316 (LISC!); Menezes, Henriques \& Brites 2892 (LISC!); Welwitsch 1446 (BM LISU!).

C. fugosioides: Antunes vel Dekindt 587 (B LISC!); Baum 174 (B†? BM COI G K M W); Faulkner 68
(BM K PRE); Gossweiler 1971 (BM); Gossweiler 2196 (BM COI LISC!); Mendes 362 (LISC!); Mendes 730 (LISC!); Mendes 753 (LISC!); Menezes 1238 (LISC!); Menezes 1254 (LISC!); Menezes 4262 (LISC!).

C. grandistipulatum: Antunes $110(\mathrm{~B} \dagger$ ?); Bester 8990 (PRE!); Fritzsche 220 (B); Mendonça 4617 (LISC!); Ramalho s.n. (B); Teixeira e Andrade 5133 (LISC!); Welwitsch 1451 (BM LISU!).

C. huillense: Exell \& Mendonça 2708 (BM COI LISC!); Torre 8747 (LISC!).

C. johannis: Gossweiler 7238 (BMpp LISC! LISUpp!); M.E.F.A. 457 (LISC!); M.E.F.A. 676 (LISC!); Monteiro \& Murta 243 (PRE!).

C. junceum subsp. jatrophoides: Anchieta 47 (LISU!); Barbosa 11164 (LISC!); Baum 278 (B†? G W); Bester 9237 (PRE!); Gossweiler 2094 (BM); Mendes 769 (LISC!); Mendes 1073 (LISC!); Mendonça 4597 (LISC!); Menezes 2170 (LISC!); Menezes 4274 (LISC!); Menezes, Barroso \& Sousa 4514 (LISC!); Monteiro e Murta 1957 (LISC!); Nolde 81 (B); Silva 228 (LISC!); Teixeira 1666 (LISC!); Teixeira \&Andrade 6657 (LISC!); Teixeira et al. 10793 (LISC!); Wellman 1546 (B); Welwitsch 1474 (B†? BM COI LISU!); Young 1006 (BM COI LISC); Young 1090 (BM); Young 1281 (BM COI LISC!).

C. luteum: Gossweiler 2355 (BM LISC!); Mendes 1898 (LISC!).

C. mendesii: Mendes 1145 (LISC!); Mendes 1164 (LISC!).

C. pendulum: Exell \& Mendonça 167 (BM COI LISC!); Exell \& Mendonça 180 (BM COI); Mendes 1001 (LISC!); Menezes 3657 (LISC!); Welwitsch 1472 (BM COI K LISU!).

C. pruriens: Castro 8 (COI); Hundt 688 (B, BM, COI); Huntley, Roberts \& Ward 88 (PRE!); Mendes 604 (LISC!); Mendes 1012 (LISC!); Mendes 1099 (LISC!); Menezes, Barroso \& Sousa 4426 (LISC!); Menezes, Barroso \& Sousa 4511 (LISC!); Menezes, Barroso \& Sousa 4629 (LISC!); Teixeira 1667 (LISC!); Torre 8405 (LISC!); Welwitsch 1454 (BM LISU!).

C. ruacanense: Bester 9218 (PRE!); Exell \& Mendonça 2769 (BM COI LISC!); Menezes, Henriques \& Brites 2815 (PRE!); Menezes, Henriques \& Brites 2943 (PRE!); Rycroft 2423 (PRE!). 
C. sp. aff. fugosioides: Menezes 3527 (PRE!).

C. sp. aff. vogelii: Welwitsch 1482 (BM LISU!).

C. stenolobum: Barros Machado ANG.I.55-288 (LISC!); Barros Machado ANG.I.55-357 (LISC!); Bester 9340 (PRE!); Couto 37 (LISC!); Dekindt 67 (B); Gossweiler 2240 (BM COI LISC!); Gossweiler 2905 (BM COI LISC!); Hundt 367 (B); Jessen 420 (B); Mendes 606 (LISC!); Mendes 1041 (LISC!); Mendes 1126 (LISC!); Mendes 1868 (LISC!); Mendes 2230 (LISC!); Menezes 1584 (LISC!); Teixeira \& Figueira 6658 (LISC!); Torre 8606 (LISC!); Torre 8613 (LISC!); Wellman 1775 (K); Welwitsch 1449 (BM COI K LISU!); Young 938 (BM COI LISC); Young 1349 (BM).

C. stipulaceum: Exell \& Mendonça 278 (COI); Gossweiler 594 (BM K); Gossweiler 6363 (COI LISU!); Gossweiler 10318 (BM COI LISC!); Nolde 523 (B BM); Welwitsch 1475 (BM LISU!); Welwitsch 1490 (B†? BM COI LISU!); Welwitsch 1471 (BM LISU!).
C. subciliatum: Exell \& Mendonça 1407 (COI). C. uter: Abreu 51 (BM COI); Bester 9116 (PRE!); Exell \& Mendonça 2182 (BM COI LISC!); Gomes e Sousa s.n. (COI); Mendes 136 (LISC!); Mendes 136a (LISC!); Mendes 145 (LISC!); Mendes 170 (LISC!); Mendes 1309 (LISC!); Menezes, Barroso \& Sousa 4828 (LISC!); Menezes \& Henriques 216 (LISC! LISU!); Pearson 2256 (K); Pimentel Teixeira s.n. (BM K); Teixeira 1716 (LISC!); Teixeira et al. 12699 (LISC!).

C. violaceo-glandulosum: Anchieta 77 (LISU!); Baum 270 (B†? BM COI G K M S); Baum 270a (B); Carrisso \& Mendonça 579 (BM COI); Couto 282 (LISC!); Faulkner 66 (BM K PRE!); Gossweiler 2100 (BM); Henriques 816 (LISC!); Henriques 817 (LISU!); Hundt 195 (B); Mendes 607 (LISC!); Mendes 1113 (LISC!); Mendes 1114 (LISC!); Menezes 1248 (LISC!); Menezes 4259 (LISC!); Teixeira 1525 (LISC!); Teixeira \& Andrade 5024 (LISC!); Teixeira \& Figueira 4910 (LISC!).

C. wittei: Barros Machado ANG.XII.54-51 (LISC!). 